

\title{
Sur la 2-cohomologie non abélienne des modèles réguliers des anneaux locaux henséliens
}

\author{
par JEAN-CLAUDE DOUAI
}

\begin{abstract}
RÉSumÉ. Soit $A$ un anneau Notherien, local, Henselien, excellent, de corps résiduel $k, k$ étant ou algébriquement clos de caractéristique 0 ou un corps fini, $X \rightarrow$ Spec $A$ un morphisme propre dont la fibre spéciale $X_{0} \rightarrow$ Spec $A$ est de dimension au plus 1. Dans ce papier, nous complètons les résultats de [1] en montrant que si $X$ est régulier et si $L$ est un $X_{e t}$-lien localement représentable par un groupe semi-simple simplement connexe, alors toutes les classes de $H^{2}\left(X_{e t}, L\right)$ sont neutres. Prenant pour $X$ un modèle régulier de $A$, nous montrons que toutes les classes de $H^{2}(K, L)$, $K=\operatorname{Frac}(A)$, sont neutres si $\operatorname{dim}(A)=2$ et $k$ algébriquement clos de caractéristique 0 . Ceci redonne certains résultats de [2].
\end{abstract}

Abstract. Let $A$ be a Notherian, local, Henselien, excellent domain with algbraically closed residue field of caracteristic 0 or finite $k, K=\operatorname{Frac}(A), X \rightarrow \operatorname{Spec} A$ a proper morphism with special fiber $X_{0} \rightarrow$ Spec $k$ of dimension at most one. Here we complete the results of [1] showing that if $X$ is regular and if $L$ is a $X_{e t}$-lien that is locally representable by a simply connected semisimple group, then all classes of $H^{2}\left(X_{e t}, L\right)$ are neutral. Taking for $X$ a regular model of $A$, we show that all classes of $H^{2}(K, L)$ are neutral if $\operatorname{dim}(A)=2$ and if $k$ is algebraically closed of caracteristic 0 . We find again some results of $[2]$.

\section{Introduction}

Soit $A$ un anneau noethérien, local, hensélien, excellent, de corps résiduel $k$. Supposons $k$ algébriquement clos de caractéristique 0 ou $k$ fini. Soit $\pi: X \rightarrow$ Spec $A$ un morphisme propre dont la fibre spéciale $X_{0} \rightarrow$ Spec $k$ est de dimension au plus un. Colliot-Thélène, Ojanguren et Parimala ont montré que, si $X$ était régulier, alors $\operatorname{Br}(X)=\operatorname{Br}\left(X_{0}\right)=0$. Ils ont aussi établi la nullité du $H^{1}(K, \tilde{G})$ où $K=\operatorname{Frac}(A)$, quand $\operatorname{dim} A=2$ et $k$ est algébriquement clos de caractéristique $0, \tilde{G}$ désignant un $K$-groupe linéaire semi-simple simplement connexe. Dans le même esprit, nous montrons que toutes les classes de $H_{\mathrm{e} t}^{2}(X, L)$ sont neutres pour tout $X_{\mathrm{e} t}$-lien $L$ localement représentable par un $X$-schéma en groupes semi-simples simplement connexes $\tilde{G}$. En appliquant ce résultat à un modèle régulier $X \operatorname{de} A$ et, en 
combinant avec un résultat de [3], on en déduit que toutes les classes de $H^{2}(K, L)$ sont aussi neutres quand $\operatorname{dim} A=2$ et $k$ algébriquement clos, de caractéristique $0 . K$ est alors un corps de type $(l l)$ au sens de [2] et ceci redonne certains résultats de Colliot-Thélène, Gilles, Parimala tels que, par exemple, la surjectivité du cobord $H^{1}(K, G) \stackrel{\delta^{1}}{\rightarrow} H^{2}(K, \mu)$ pour $G$ semisimple et $\mu$ le noyau du revêtement universel de $G$. Ces derniers imposent toujours la condition « l'indice d'une $K$-algèbre simple centrale quelconque est égal à son exposant ». Notre résultat local de [3] donne automatiquement cette égalité quand on travaille sur les corps de type $(s l)$ au sens de [2] (cf. aussi le théorème 1.5 de [2]) et, de là par globalistion, sur les corps de type de type $(l l)$ (cf. aussi le théorème 1.3 de [2]).

Je tiens à remercier le referee pour son aide et ses corrections.

Dans toute la suite, nous supposerons $A$ noethérien.

\section{$\S I$.}

Proposition 1.1. Soient $A$ un anneau local hensélien, $k$ son corps résiduel, $\pi: X \rightarrow$ Spec $A$ un morphisme propre, $X_{0} \rightarrow$ Spec $k$ la fibre de $\pi$ au-dessus du point fermé. Soit $L$ un lien localement (pour la topologie étale) représentable par un $X$-groupe semi-simple $G$. [On sait qu'alors $L$ est représentable par un $X$-groupe $G_{L}$ de même type que $G$ (cf. par ex. la prop. 1.1 de [4] $-G_{L}$ peut même être pris quasi-déployé)]. Alors, désignant par $Z\left(G_{L}\right)$ le centre de $G_{L}$ (resp. par $Z(L)$ le centre de $L$ ), l'indice 0 indiquant la restriction à $X_{0}$,

$$
H_{e t}^{2}(X, Z(L))=H_{e t}^{2}\left(X, Z\left(G_{L}\right)\right) \stackrel{\sim}{\rightarrow} H_{e t}^{2}\left(X_{0}, Z\left(G_{L_{0}}\right)\right)
$$

et $H_{\text {et }}^{2}(X, L)$ est isomorphe à $H_{\text {et }}^{2}\left(X_{0}, L_{0}\right)$, cet isomorphisme d'ensembles étant compatible avec les actions simplement transitives respectives de $H_{\text {et }}^{2}\left(X, Z\left(G_{L}\right)\right)$ et $H_{\text {et }}^{2}\left(X_{0}, Z\left(G_{L_{0}}\right)\right)$.

Démonstration. $Z\left(G_{L}\right)$ est un $X$-faisceau en groupes finis et l'isomorphisme

$$
H_{\text {et }}^{2}\left(X, Z\left(G_{L}\right)\right) \stackrel{\sim}{\rightarrow} H_{e t}^{2}\left(X_{0}, Z\left(G_{L_{0}}\right)\right)
$$

résulte du théorème de changement de base propre (cf. par exemple [6] - VI - 2.7). Or, $H_{e t}^{2}\left(X, Z\left(G_{L}\right)\right)$ (resp. $H_{e t}^{2}\left(X_{0}, Z\left(G_{L_{0}}\right)\right)$ opère simplement transitivement sur $H_{e t}^{2}(X, L)$ (resp. $H_{e t}^{2}\left(X_{0}, L_{0}\right)$ ) (cf. le théor. 3.3.3 - Chap. IV de $[6])$. Compte tenu du fait que $H_{e t}^{2}(X, L)$ (resp. $\left.H_{e t}^{2}\left(X_{0}, L\right)\right)$ est pointé par la classe neutre unité $\varepsilon=\left[\operatorname{Tors}\left(X_{e t}, G_{L}\right)\right]\left(\operatorname{resp} . \varepsilon_{0}=\left[\operatorname{Tors}\left(X_{0, e t}, G_{L_{0}}\right)\right]\right)$, on obtient alors le résultat.

Conjecture 1.2. Sous les hypothèses de la prop. 1.1, il y a autant de classes dans $H_{e t}^{2}(X, L)$ que dans $H_{e t}^{2}\left(X_{0}, L_{0}\right)$. Il nous faut alors comparer les sous-ensembles des classes neutres de $H_{e t}^{2}\left(X, G_{L}\right)$ et $H_{e t}^{2}\left(X_{0}, G_{L_{0}}\right)$ : on 
peut conjecturer que l'image inverse par l'application

$$
\begin{array}{cc}
H_{e t}^{2}(X, L) & H_{e t}^{2}\left(X_{0}, L_{0}\right) \\
\| & \| \\
H_{e t}^{2}\left(X, G_{L}\right) & H_{e t}^{2}\left(X_{0}, G_{L_{0}}\right)
\end{array}
$$

de l'ensemble des classes neutres de $H_{e t}^{2}\left(X_{0}, L_{0}\right)$ est l'ensemble des classes neutres de $H_{e t}^{2}(X, L)$ étendant ainsi le résultat du cor. 2.2.6 (ii) du chap. VII de [5] où le résultat est énoncé pour les liens ind-finis $L$. On sait seulement que l'application :

$$
H_{e t}^{1}\left(X,\left(G_{L}\right)_{a d}\right) \longrightarrow H_{e t}^{1}\left(X_{0},\left(G_{L_{0}}\right)_{a d}\right)
$$

est injective (cf. cor.5.5-exposé XII, p.90, Lecture Notes $\mathrm{n}^{\circ} 305$ ).

Dans le théorème 1.3 suivant, nous montrons que, dans le cas où la fibre spéciale est de dimension au plus $1, k$ étant séparablement clos ou fini et $G=\tilde{G}$ semi-simpe simplement connexe, alors toutes les classes de $H_{e t}^{2}(X, L)$ sont neutres, toutes les classes de $H_{e t}^{2}\left(X_{0}, L_{0}\right)$ étant neutres, ce qui établit la conjecture 1.2 dans ce cas.

Théorème 1.3. Soient $A$ un anneau local hensélien et $k$ son corps résiduel. Supposons $k$ soit ou séparablement clos ou fini. Soit $\pi: X \rightarrow$ Spec $A$ un morphisme propre dont la fibre spéciale $X_{0} \rightarrow$ Spec $k$ est de dimension au plus 1. Supposons $X$ régulier. Soit $\tilde{G}$ un $X$-groupe semi-simple simplement

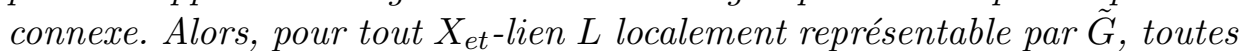
les classes de $H_{\text {et }}^{2}(X, L)$ sont neutres.

Démonstration. Le lien $L$ est représentable par un $X$-groupe semi-simple, simplement connexe, quasi-déployé $\tilde{G}_{L}: \tilde{G}_{L}$ admet un $X$-couple de Killing $(\tilde{B}, \tilde{T})$ où $\tilde{T}$ est un $X$-tore induit (cf. la prop. 3.13 - exposé XXIV de S.G.A.D. [7]) :

$$
\tilde{T}=\prod_{X^{\prime} / X} G_{m_{X^{\prime}}},
$$

$X^{\prime}$ ouvert étale fini de $X, \prod_{X^{\prime} / X}$ désignant la restriction à la Weil de $X^{\prime}$ à $X$, d'où

$$
H^{2}(X, \tilde{T})=H_{e t}^{2}(X, \tilde{T})=H_{e t}^{2}\left(X^{\prime}, G_{m}\right)=\operatorname{Br}\left(X^{\prime}\right)=\operatorname{Br}\left(X_{0}^{\prime}\right)=0
$$

(cf. par ex. le corollaire 1.10 (b) et le corollaire 1.11 (b) de [1]). Donc $H^{2}(X, \tilde{T})=0$. 
- Soit $q$ une classe de $H_{e t}^{2}(X, L)=H_{e t}^{2}\left(X, \tilde{G}_{L}\right)$. Ecrivons $q$ sous la forme $\alpha \cdot \varepsilon$ où $\alpha \in H^{2}\left(X, Z\left(\tilde{G}_{L}\right)\right), \varepsilon=$ classe « unité » [Tors $\left.\tilde{G}_{L}\right]$ de $H_{e t}^{2}\left(X, \tilde{G}_{L}\right)$. La classe $q$ est en relation avec $\alpha$ par

$$
\begin{array}{ccc}
H_{e t}^{2}\left(X, Z\left(\tilde{G}_{L}\right)\right) & \longrightarrow & H_{e t}^{2}\left(X, \tilde{G}_{L}\right) \\
\alpha & \multimap & q .
\end{array}
$$

Mais $q$ est aussi en relation avec l'image de $\alpha$ dans $H^{2}(X, \tilde{T})=0$ par la relation

$$
H^{2}(X, \tilde{T}) \multimap H_{e t}^{2}\left(X, \tilde{G}_{L}\right)
$$

induite par $\tilde{T} \hookrightarrow \tilde{G}_{L}$ (cf. la déf. 3.1.4 - Chap. IV de [5] pour la relation $\longrightarrow$ ) dans le diagramme suivant :

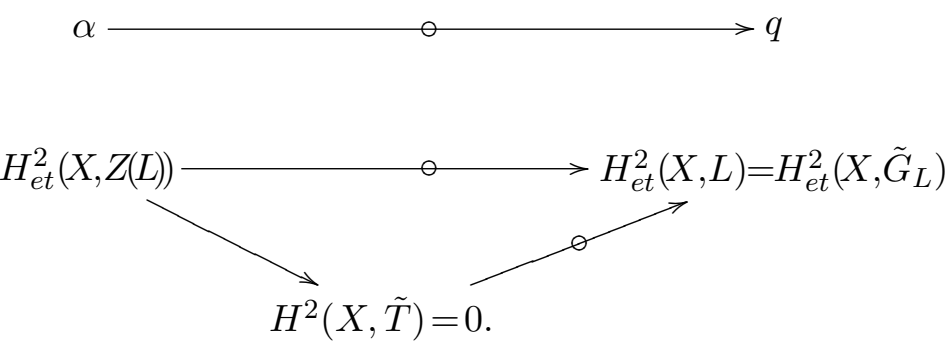

- La classe $q$ est donc nécessairement neutre, ce qui démontre le théorème.

Corollaire 1.4 (au théorème 1.3).

$$
H_{e t}^{2}(X, L)=H_{e t}^{2}(X, L)^{\prime} \simeq H_{e t}^{2}\left(X_{0}, L_{0}\right)^{\prime}=H_{e t}^{2}\left(X_{0}, L_{0}\right)
$$

où le' désigne le sous-ensemble des classes neutres de $H_{e t}^{2}(X, L)$ (resp. $\left.H_{\text {et }}^{2}\left(X_{0}, L_{0}\right)\right)$.

\section{$\S$ II.}

Dans cette deuxième partie, nous appliquerons les résultats du $\S$ I précédent au cas où $A$ est un anneau local, hensélien, excellent, de dimension 2 , de corps résiduel $k$ algébriquement clos et de caractéristique 0 . Selon Hironaka, Abhyankar et Lipman, il existe alors des modèles réguliers $X$ de $A$ i.e. des schémas réguliers $X$ munis d'un morphisme projectif birationnel $X \rightarrow S p e c A$. La fibre $X_{0}$ de $X \rightarrow \operatorname{Spec} A$ en le point fermé de Spec $A$ est une variété projective de dimension au plus 1 sur $k$. La propriété que $k$ est algébriquement clos de caractéristique 0 implique que $c d \cdot(K)$, où $K=\operatorname{Frac}(A)$, est égal à 2 (si $k$ était de caractéristique $p$, on aurait seulement $c d_{l}(K)=2$ pour $\left.l \neq p\right)$.

- Supposons donc que $A$ est un anneau local, de dimension 2, hensélien, excellent, à corps résiduel $k$ algébriquement clos, de caractéristique 0 . Le corps $K=\operatorname{Frac}(A)$ est un corps de type $(l l)=($ local $/$ local $)$ comme défini dans [2]. Pour $X$ un modèle projectif lisse de $A$, soient $X^{1}$ l'ensemble des 
points de codimension 1 de $X$ et $\Omega_{X}$ l'ensemble des valuations discrètes $v$ associées aux points $x \in X^{1}$. Soit $\Omega$ la réunion sur tous les modèles projectifs lisses $X$ des $\Omega_{X}$. Par le corollaire 1.10 de [1], $\operatorname{Br}(X)=0$. La nullité de $B r(X)$ implique la trivialité de $B r_{n r}(K)$ défini à la page 187 dans loc-citado où $B r_{n r}(K)$ désigne le groupe de Brauer non ramifié de $K$ consistant de toutes les classes de $\operatorname{Br}(K)$ qui sont non ramifiées en toute valuation discrète de $K$. La trivialité de $B r_{n r}(K)$ implique à son tour la trivialité du noyau de la fonction restriction diagonale des groupes de Brauer

$$
\operatorname{Br}(K) \longrightarrow \bigoplus_{v \in \Omega_{X}} \operatorname{Br}\left(K_{v}\right)
$$

et, a fortiori, du noyau de

$$
\operatorname{Br}(K) \longrightarrow \prod_{v \in \Omega} \operatorname{Br}\left(K_{v}\right)
$$

- On sait par [2] p. 293 que chaque $K_{v}$, pour $v \in \Omega$, est un corps de type $(s l)=($ semi - local $)$. En utilisant la théorie de Bruhat-Tits, il a été montré dans [3] Chap. VII - Théorème 3.1, p. 99 (cf aussi les corollaires 2.6 et 2.8 de [4]) que si $G=\tilde{G}$ est un groupe semi-simple simplement connexe défini sur un corps semi-local $K_{v}$, alors toutes les classes de $H^{2}\left(K_{v}, L\right)$, où $L$ est un $K_{v}$-lien localement (pour la topologie étale) représentable par $\tilde{G}$, sont neutres. Ce résultat implique, en particulier, l'égalité entre l'indice et l'exposant pour les corps de type $(s l)$, mentionnée dans l'introduction. Nous obtenons alors :

Théorème 2.1. Soit $A$ un anneau local, hensélien, excellent, de dimension 2 , de corps résiduel $k$ algébriquement clos de caractéristique $0, K$ son corps des fractions, $L$ un $K$-lien localement (pour la topologie étale) représentable par un $K$-groupe semi-simple simplement connexe $\tilde{G}=\tilde{G}_{L}$. Alors toutes les classes de $H^{2}(K, L)$ sont neutres.

Démonstration. Dans la démontration du théorème 1.3, nous avons considéré la relation

$$
\begin{aligned}
& H^{2}(X, \tilde{T})=0 \quad \longrightarrow \quad H_{e t}^{2}\left(X, \tilde{G}_{L}\right)=H_{e t}^{2}(X, L) \\
& \quad \operatorname{Br}\left(X^{\prime}\right)
\end{aligned}
$$

où $X^{\prime}$ était défini par la condition $\tilde{T} \simeq \prod_{X^{\prime} / X} G_{m_{X^{\prime}}}$.

Par ce qui a été dit précédemment, la flèche

$$
\begin{gathered}
\operatorname{Br}\left(K^{\prime}\right) \hookrightarrow \bigoplus_{v \in \Omega_{X^{\prime}}} H^{2}\left(K_{v}^{\prime}, G_{m}\right) \\
\left(K^{\prime}=\text { corps des fonctions de } X^{\prime}\right)
\end{gathered}
$$

est injective. Elle s'insère dans le diagramme suivant : 


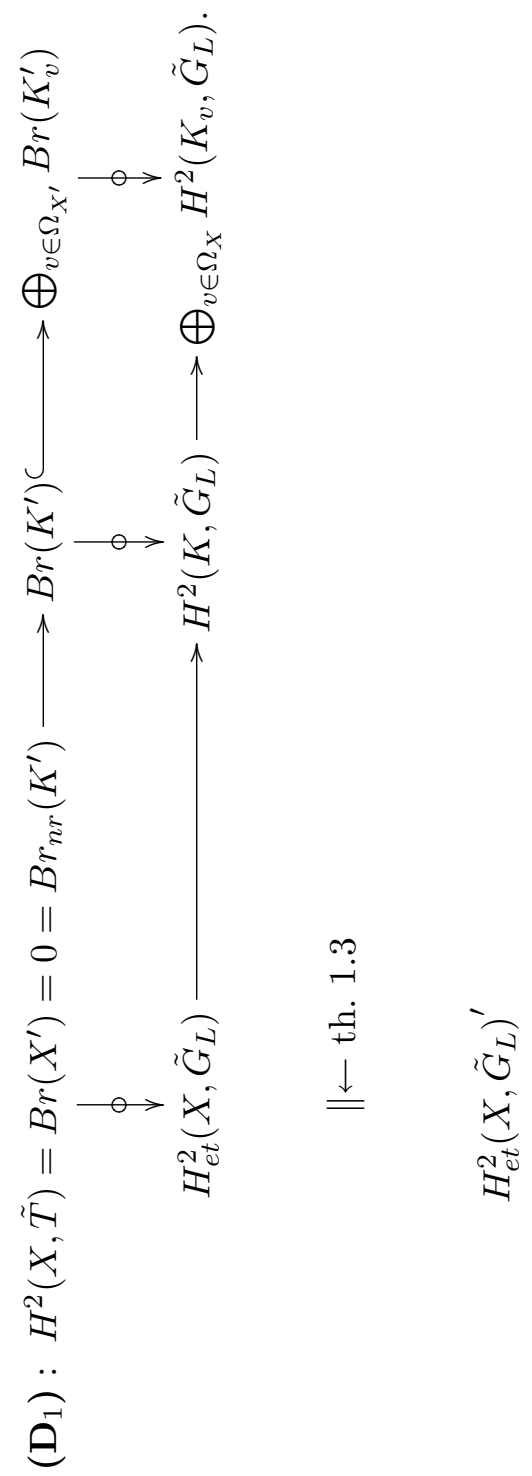


- L'application

$$
H^{2}\left(K, \tilde{G}_{L}\right) \rightarrow \bigoplus_{v \in \Omega_{X}} H^{2}\left(K_{v}, \tilde{G}_{L}\right)
$$

est compatible avec les actions respectives de $H^{2}\left(K, Z\left(\tilde{G}_{L}\right)\right)$ et $\bigoplus_{v \in \Omega_{X}} H^{2}\left(K_{v}, Z\left(\tilde{G}_{L}\right)\right)$. L'injectivité de

$$
B r\left(K^{\prime}\right) \hookrightarrow \bigoplus_{v \in \Omega_{X^{\prime}}} B r\left(K_{v}^{\prime}\right)
$$

implique immédiatement l'injectivité de

$$
H^{2}\left(K^{\prime}, \mu_{n}\right) \rightarrow \bigoplus_{v \in \Omega_{X^{\prime}}} H^{2}\left(K_{v}^{\prime}, \mu_{n}\right) \quad \forall n \geq 0,
$$

puis celle de

$$
H^{2}\left(K, Z\left(\tilde{G}_{L}\right)\right) \rightarrow \bigoplus_{v \in \Omega_{X}} H^{2}\left(K_{v}, Z\left(\tilde{G}_{L}\right)\right) .
$$

L'application

$$
H^{2}\left(K, \tilde{G}_{L}\right) \rightarrow \oplus_{v \in \Omega_{X}} H^{2}\left(K_{v}, \tilde{G}_{L}\right)
$$

dans le diagramme $\left(\mathbf{D}_{1}\right)$ est donc injective (comme application d'ensembles). En particulier, si une classe de $H^{2}\left(K, \tilde{G}_{L}\right)$ est envoyée sur la classe unité $\bigoplus_{v \in \Omega_{X}} \epsilon_{v}=\bigoplus_{v \in \Omega_{X}}\left[\operatorname{Tors}\left(\tilde{G}_{L}\right)_{v}\right]$ de $\bigoplus_{v \in \Omega_{X}} H^{2}\left(K_{v}, \tilde{G}_{L}\right)$, alors elle coincide avec la classe unité $\epsilon=\left[\right.$ Tors $\left.\tilde{G}_{L}\right]$.

Montrons maintenant que toutes les classes de $H^{2}\left(K, \tilde{G}_{L}\right)$ sont neutres. Nous en donnons deux démonstrations.

Considerons le diagramme suivant : 


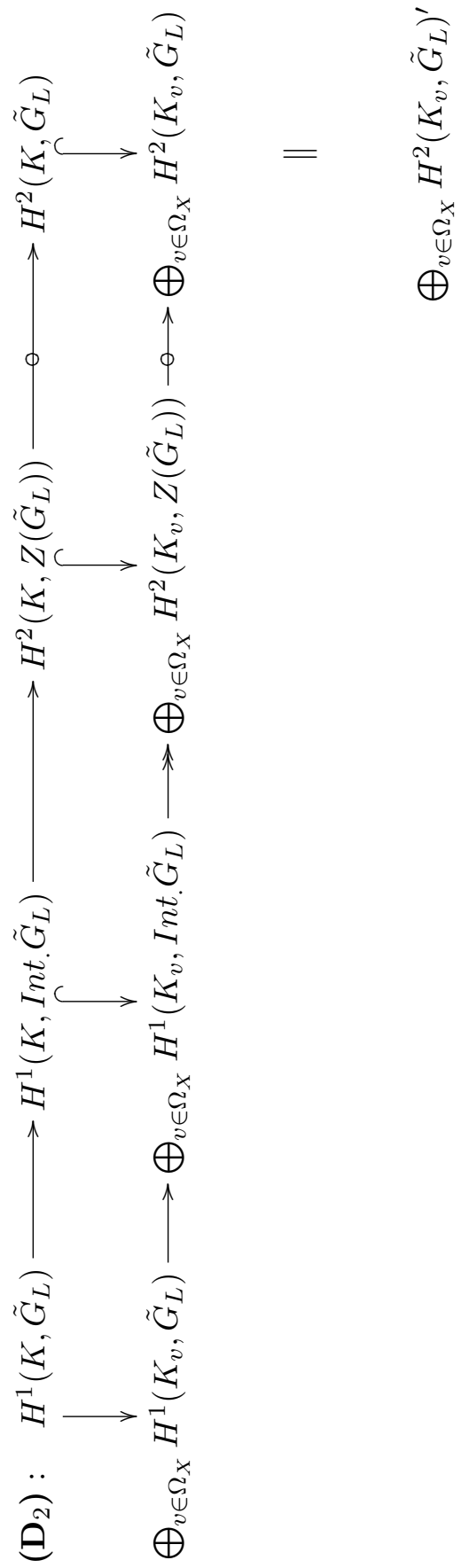


La deuxième flèche verticale est injective par le théorème 5.3 de [1] (propriété non indipensable dans la suite).

Dans le diagramme $\left(\mathbf{D}_{2}\right)$, toutes les classes de $\bigoplus_{v \in \Omega_{X}} H^{2}\left(K_{v}, Z\left(\tilde{G}_{L}\right)\right)$ sont envoyées sur la classe unité $\bigoplus_{v \in \Omega_{X}} \epsilon_{v}$ de $\bigoplus_{v \in \Omega_{X}} H^{2}\left(K_{v}, \tilde{G}_{L}\right)$ par la relation $\longrightarrow$ dans la deuxième ligne. Du carré de droite dans le diagramme $\left(\mathbf{D}_{2}\right)$ résulte alors que toutes les classes de $H^{2}\left(K, Z\left(\tilde{G}_{L}\right)\right)$ sont envoyées sur la classe $\epsilon=\left[\right.$ Tors $\left.\tilde{G}_{L}\right]$ par la relation $\longrightarrow$ de la première ligne, d'où la surjectivité de l'application

$$
H^{1}\left(K, \text { Int. } \tilde{G}_{L}\right) \longrightarrow H^{2}\left(K, Z\left(\tilde{G}_{L}\right)\right)
$$

dans la suite exacte d'ensemble pointés

$$
H^{1}\left(K, \tilde{G}_{L}\right) \longrightarrow H^{1}\left(K, \text { Int. } \tilde{G}_{L}\right) \longrightarrow H^{2}\left(K, Z\left(\tilde{G}_{L}\right)\right) \longrightarrow 1
$$

Comparant avec la suite exacte :

$$
H^{1}\left(K, \tilde{G}_{L}\right) \longrightarrow H^{1}\left(K, \text { Int } \tilde{G}_{L}\right) \longrightarrow H^{2}\left(K, \tilde{G}_{L}\right)^{\prime} \longrightarrow 1
$$

de la proposition 3.2.6(iii)-Chap.IV de [5], on en déduit que $H^{2}\left(K, \tilde{G}_{L}\right)=$ $H^{2}\left(K, \tilde{G}_{L}\right)^{\prime}$,i.e que toute classe de $H^{2}\left(K, \tilde{G}_{L}\right)$ est neutre.

Plus rapidement, on peut dire que la classe 0 de $H^{2}\left(K_{v}, Z\left(\tilde{G}_{L}\right)\right)$, pour $v \in \Omega_{X}$, est en relation avec toute classe de $H^{2}\left(K_{v}, \tilde{G}_{L}\right)=H^{2}\left(K_{v}, \tilde{G}_{L}\right)^{\prime}$ (cf par ex. le corollaire 3.3.7 (i) Chap.IV de [5]). D'où la classe $(0,0, \cdots)$ de $\bigoplus_{v} H^{2}\left(K_{v}, Z\left(\tilde{G}_{L}\right)\right)$ est en relation avec toute classe de $\bigoplus_{v} H^{2}\left(K_{v}, \tilde{G}_{L}\right)$. Par le carré de droite du diagramme $\left(\mathbf{D}_{2}\right)$, la classe 0 de $H^{2}\left(K, Z\left(\tilde{G}_{L}\right)\right)$ est donc en relation avec toute classe de $H^{2}\left(K, \tilde{G}_{L}\right)$, i.e toute $K$-gerbe à lien représentable par $\tilde{G}_{L}$ admet une section, i.e toutes les classes de $H^{2}\left(K, \tilde{G}_{L}\right)$ sont neutres.

Corollaire 2.2. Toutes les classes de

$$
H_{e t}^{2}\left(X, \tilde{G}_{L}\right)^{\prime}=H_{e t}^{2}\left(X, \tilde{G}_{L}\right)
$$

sont envoyées sur la classe unité $\left[\right.$ Tors $\left.\tilde{G}_{L}\right]$ de $H^{2}\left(K, \tilde{G}_{L}\right)$ par l'application $H_{e t}^{2}\left(X, \tilde{G}_{L}\right) \rightarrow H^{2}\left(K, \tilde{G}_{L}\right)$.

- L'application $H_{e t}^{2}\left(X, \tilde{G}_{L}\right) \rightarrow H^{2}\left(K, \tilde{G}_{L}\right)$ est compatible avec les actions de $H_{e t}^{2}\left(X, Z\left(\tilde{G}_{L}\right)\right)\left(\operatorname{resp} . H^{2}\left(K, Z\left(\tilde{G}_{L}\right)\right)\right)$ sur $H_{e t}^{2}\left(X, \tilde{G}_{L}\right)\left(\operatorname{resp.} H^{2}\left(K, \tilde{G}_{L}\right)\right)$. Supposons, pour simplifier, que $Z\left(\tilde{G}_{L}\right)=\mu_{n}, n \geq 0$. L'homomorphisme $H_{e t}^{2}\left(X, \mu_{n}\right) \rightarrow H^{2}\left(K, \mu_{n}\right)$ se factorise par $B r(X)_{n}$ de la manière suivante : 


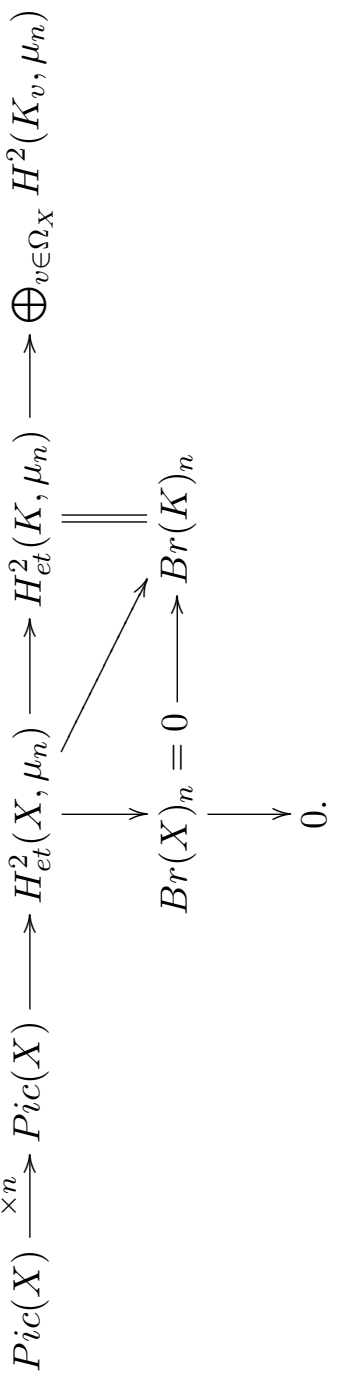


Il est donc nul : dans le cas où $Z\left(\tilde{G}_{L}\right)=\mu_{n}$, toutes les classes de $H_{e t}^{2}\left(X, \tilde{G}_{L}\right)=H^{2}\left(X, \tilde{G}_{L}\right)^{\prime}$ sont envoyées sur la classe unité de $H^{2}\left(K, \tilde{G}_{L}\right)$. On se ramène ensuite au cas où $Z\left(\tilde{G}_{L}\right)=\mu_{n}$.

Corollaire 2.3. Supposons que $A$ et $K$ sont comme dans le théorème 2.1. Si $L$ est un $K$-lien localement (pour la topologie étale) représentable par un $K$-groupe semi-simple, toutes les classes de $H^{2}(K, L)$ sont neutres.

En effet, $L$ est représentable par un $K$-groupe semi-simple $G_{L}$. Soit $\tilde{G}_{L}$ un revêtement universel de $G_{L}$ :

$$
1 \rightarrow \mu \rightarrow \tilde{G}_{L} \rightarrow G_{L} \rightarrow 1 \text {. }
$$

Comme $K$ est de dimension cohomologique 2, l'application

$$
H^{2}\left(K, \tilde{G}_{L}\right) \rightarrow H^{2}\left(K, G_{L}\right)
$$

est surjective. Toutes les classes de $H^{2}\left(K, G_{L}\right)$ sont donc neutres. c.q.f.d.

Le corollaire 2.3 précédent redonne en particulier la proposition 5.3 de [2] pour les corps de type $(l l)$.

\section{Bibliographie}

[1] J.L. Colliot-Thélène, M. Ojanguren and R. Parimala, Quadratic forms over fraction fields of two-dimensional Henselian rings and Brauer groups of related schemes. In Algebra, Arithmetic and Geometry, I, II (Mumbai, 2000), Tata Inst. Fund. Res. Stud. Math. 16 (2002), 185-217. MR 1940669.

[2] J.L. Colliot-Thélène, P. Gille and R. Parimala, Arithmetic of linear algebraic groups over 2-dimensional geometric fields. Duke Math. J. 121 (2004), 285-341.

[3] J.C. DouAI, 2-cohomologie galoisienne des groupes semi-simples. Thèse d'Etat, Université de Lille 1, Lille, France, 1976.

[4] J.C. DouAI, Sur la 2-cohomologie galoisienne de la composante résiduellement neutre des groupes réductifs connexes définis sur les corps locaux. C.R. Acad. Sci. Paris, Série I 342 (2006), 813-818.

[5] J. Giraud, Cohomologie non abélienne. Grundlheren Math. Wiss. vol. 179, Springer-Verlag, 1971.

[6] J.S. Milne, Etale cohomology. Princeton Mathematical Series, vol. 33, Princeton University Press, Princeton, 1980.

[7] S.G.A.D., Séminaire de géométrie algébrique 1963-1964. Lecture Notes in Math., 151-153, Springer, 1970.

Jean-Claude DouAI

Laboratoire Painlevé

U.M.R 8524

Université des sciences et technologies de Lille 1

59655 Villeneuve d'ascq cedex France

E-mail: douai@math.univ-lille1.fr 